Article

\title{
Euler Sums and Integral Connections
}

\author{
Anthony Sofo ${ }^{1, *}$ and Amrik Singh Nimbran ${ }^{2}$ (D) \\ 1 College of Engineering and Science, Victoria University, P. O. Box 14428, Melbourne City, \\ Victoria 8001, Australia \\ 2 B3-304, Palm Grove Heights, Ardee City, Gurugram, Haryana 122003, India \\ * Correspondence: anthony.sofo@vu.edu.au
}

Received: 13 August 2019; Accepted: 4 September 2019; Published: 9 September 2019

\begin{abstract}
In this paper, we present some Euler-like sums involving partial sums of the harmonic and odd harmonic series. First, we give a brief historical account of Euler's work on the subject followed by notations used in the body of the paper. After discussing some alternating Euler sums, we investigate the connection of integrals of inverse trigonometric and hyperbolic type functions to generate many new Euler sum identities. We also give some new identities for Catalan's constant, Apery's constant and a fast converging identity for the famous $\zeta(2)$ constant.
\end{abstract}

Keywords: Euler sums; Catalan's constant; Trigamma function; integral representation; closed form; ArcTan and ArcTanh functions; partial fractions

\section{Historical Background and Preliminaries}

\subsection{Euler's Work}

We begin by touching on the historical background of Euler sums. The 20th century British mathematician G. H. Hardy remarked in A Mathematician's Apology (1940): “'Immortality' may be a silly word, but probably a mathematician has the best chance of whatever it may mean." Leonhard Euler (1707-1783), the versatile and prolific Swiss mathematician, achieved immortality through his pioneering work on infinite series and products which began when he was 22 . He gained instant fame in youth by solving the Basel problem (suggested to him by Johann Bernoulli who had tried and failed) which asked for the exact sum of the series of reciprocals of squares of natural numbers. He generalized the problem and introduced the famous Euler zeta function: $\sum_{n=1}^{\infty} \frac{1}{n^{m}}, m \geq 2$. Euler extended the concept of factorial to positive rational numbers. His investigation (motivated by Christian Goldbach, the secretary of the Petersburg Academy where Euler worked) laid the foundation for another famous function, the gamma function, the digamma function (the logarithmic derivative of the gamma function) and the polygamma functions formed by repeated differentiation of the digamma function. Nicholas Oresme (1323-1382) had showed that the sum $\sum_{k=1}^{\infty} \frac{1}{k}$ diverges. Euler investigated its partial sums (called harmonic numbers), $\sum_{k=1}^{n} \frac{1}{k}$, detected their link with logarithms, and introduced his ubiquitous constant $\gamma$ in the process: $\gamma=\lim _{n \rightarrow \infty}\left\{H_{n}-\log (n)\right\}$, where $n$ is any positive integer. He also gave the formula: $H_{n}=\int_{0}^{1} \frac{1-x^{n}}{1-x} d x$ which can be established by writing the integrand as a geometric progression and then integrating it term by term. 
Euler sums have their origin in the correspondence between Goldbach and Euler after Euler's departure from Russia in 1741. In his letter of 6 December 1742, Goldbach informed Euler that he had calculated the sums of the following series ([1] p. 741):

$$
\begin{gathered}
1-\frac{1}{2}\left(1 \mp \frac{1}{2}\right)+\frac{1}{3}\left(1 \mp \frac{1}{2}+\frac{1}{3}\right)-\frac{1}{4}\left(1 \mp \frac{1}{2}+\frac{1}{3} \mp \frac{1}{4}\right)+\ldots, \\
1-\frac{1}{2}\left(1-\frac{1}{2^{2}}\right)+\frac{1}{3}\left(1-\frac{1}{2^{2}}+\frac{1}{3^{2}}\right)-\frac{1}{4}\left(1-\frac{1}{2^{2}}+\frac{1}{3^{2}}-\frac{1}{4^{2}}\right)+\ldots, \\
\frac{1}{2^{2}}(1)-\frac{1}{3^{2}}\left(1-\frac{1}{2}\right)+\frac{1}{4^{2}}\left(1-\frac{1}{2}+\frac{1}{3}\right)-\frac{1}{5^{2}}\left(1-\frac{1}{2}+\frac{1}{3}-\frac{1}{4}\right)+\ldots
\end{gathered}
$$

However, in his next letter (24th December), he modified his claims stating that they arose out of an error which led to serendipitous discovery of Euler sums:

when I recently reconsidered the supposed sums of the two series mentioned at the end of my last letter, I perceived at once that they had arisen by a mere writing mistake. However, of this indeed the proverb says "If he had not erred, he should have achieved less"; for on that occasion I came upon the summations of some other series which otherwise I should hardly have looked for, much less discovered.

Goldbach then ([1] p. 747) proposed to compute the sum:

$$
1+\frac{1}{2^{n}}\left(1+\frac{1}{2^{m}}\right)+\frac{1}{3^{n}}\left(1+\frac{1}{2^{m}}+\frac{1}{3^{m}}\right)+\ldots
$$

where $m, n$ are not equal even integers. He further gave the sum $\frac{\pi^{4}}{72}$ for series with $m=1, n=3$. This led Euler to investigate this sum. In his reply of 5 January 1743 to Goldbach, he recorded various sums including ([1] p. 752, G, I):

$$
1-\frac{1}{2}\left(1+\frac{1}{2}\right)+\frac{1}{3}\left(1+\frac{1}{2}+\frac{1}{3}\right)-\frac{1}{4}\left(1+\frac{1}{2}+\frac{1}{3}+\frac{1}{4}\right)+\cdots=\frac{\pi^{2}}{12}-\frac{\log ^{2}(2)}{2},
$$

and

$$
1-\frac{1}{2}\left(1-\frac{1}{2}\right)+\frac{1}{3}\left(1-\frac{1}{2}+\frac{1}{3}\right)-\frac{1}{4}\left(1-\frac{1}{2}+\frac{1}{3}-\frac{1}{4}\right)+\cdots=\frac{\pi^{2}}{12}+\frac{\log ^{2}(2)}{2}
$$

which appeared in $\$ 108$ (page 509) of his Continuatio Fragmentorum ex Adversariis mathematicis depormtorum (Opera Postuma 1, 1862, 487-518) now numbered E819.

Their correspondence culminated in a 47-page paper [2] by Euler (1776) using a cumbersome notation:

$$
\int \frac{1}{z^{m}}\left(\frac{1}{y^{n}}\right)=1+\frac{1}{2^{m}}\left(1+\frac{1}{2^{n}}\right)+\frac{1}{3^{m}}\left(1+\frac{1}{2^{n}}+\frac{1}{3^{n}}\right)+\ldots
$$

In the paper mentioned, he employed three methods (which he called Prima Methodus, Secunda Methodus and Tertia Methodus) to discover formulas representing these sums in terms of zeta values. First, he multiplied the involved series to obtain this formula (§4, p.144):

$$
\int \frac{1}{z^{m}}\left(\frac{1}{y^{n}}\right)+\int \frac{1}{z^{n}}\left(\frac{1}{y^{m}}\right)=\int \frac{1}{z^{m}} \int \frac{1}{z^{n}}+\int \frac{1}{z^{m+n}} .
$$


After investigating cases with combined exponents of $z, y=2,3, \ldots, 10$, he considered the sum with $n=1$ in the notation given earlier. He recorded a general formula (\$22, p. 165) which, for $m=2,3,4, \ldots$, can be written as:

$$
E U(m)=\sum_{n=1}^{\infty} \frac{H_{n}}{n^{m}}=\frac{m+2}{2} \zeta(m+1)-\frac{1}{2} \sum_{k=1}^{m-2} \zeta(m-k) \zeta(k+1) .
$$

\subsection{Post-Euler Development}

Nielsen (1906) built on and supplemented Euler's work by supplying proof of the general Formula (1) using the method of partial fractions in ([3] pp. 47-49). Ramanujan noted a few Euler sums in his notebooks sometime in the 1910s. Sitaramachandrarao, an Indian mathematician, obtained identities for various alternating Euler sums in 1987 while discussing a formula of Ramanujan [4].

Georghiou and Philippou [5] established Euler's formula as well as:

$$
\sum_{n=1}^{\infty} \frac{H_{n}}{n^{2 s+1}}=\frac{1}{2} \sum_{r=2}^{2 s}(-1)^{r} \zeta(r) \zeta(2 s+2-r), \quad s \geq 1 .
$$

They also gave this formula:

$$
\begin{aligned}
\sum_{n=1}^{\infty} \frac{H_{n}^{(2)}}{n^{2 s+1}} & =\zeta(2) \zeta(2 s+1)-\frac{(s+2)(2 s+1)}{2} \zeta(2 s+3) \\
& +2 \sum_{r=2}^{s+1}(r-1) \zeta(2 r-1) \zeta(2 s+4-2 r), \quad s \geq 1
\end{aligned}
$$

where $H_{n}^{(m)}=\sum_{\ell=1}^{n} \ell^{-m}, m \geq 1, n \geq 1$. They remarked at the end that it was still an open question to give a closed form of $\sum_{n=1}^{\infty} \frac{H_{n}^{(p)}}{n^{q}}$ for any integers $p \geq 1$ and $q \geq 2$ in terms of the zeta function. Surprisingly, they did not mention Euler or his work even once.

The publication of De Doelder's paper [6] in 1991 and three papers submitted during July, August and October 1993 by the Borweins and their co-researchers [7-9] produced a revival of interest in these sums among a number of mathematicians. Crandall and Buhler [10] established various series expansions of $\sum_{n=2}^{\infty} \frac{1}{n^{s}} \sum_{m=1}^{n-1} \frac{1}{m^{r}}$. Various approaches have been employed to get such sums. De Doelder summed some series by evaluating integrals and using the digamma function. A quadratic $\operatorname{sum} \sum_{k=1}^{\infty} \frac{\{\psi(k)-\psi(1)\}^{2}}{k^{2}}=\sum_{n=1}^{\infty}\left(\frac{H_{n}}{n+1}\right)^{2}=\frac{11 \pi^{4}}{360}$ was derived by De Doelder and an associated sum conjectured by Enrico Au-Yeung (an under-graduate student at Waterloo, ([7] p. 17) and ([8] p. 1191)) on the basis of his computations was established by means of generating functions and Parseval's identity for Fourier series by the Borweins. In fact, the associated sum $\sum_{n=1}^{\infty}\left(\frac{H_{n}}{n}\right)^{2}=\frac{17 \pi^{4}}{360}$ had been computed by Sandham [11] in 1948, but it remained generally unknown. Chu [12] made use of the hypergeometric method for deriving some Euler sums. Jung, Cho and Choi [13] use integrals from Lewin's book to evaluate different Euler sums. In particular, they show that $\sum_{n=1}^{\infty} \frac{H_{n}}{(n+1)^{3}}=\frac{\zeta(4)}{4}$ can be obtained from the integral (7.65) recorded in ([14] p. 204). Flajolet and Salvy [15] used contour integration. Freitas [16] transformed De Doelder's sum into a double integral and then evaluated the integral directly. Experimental evaluation of Euler sums involving heavy use of Maple and Mathematica led to the discovery of many new sums; some of these were established later. Euler sums have been a popular topic for engaging many mathematicians for last many years. 


\subsection{Notations and Representations of Harmonic Numbers}

We use the following notations throughout our paper:

1. $H_{n}=\sum_{k=1}^{n} \frac{1}{k} ; \quad H_{n}^{\prime}=\sum_{k=1}^{n} \frac{(-1)^{k+1}}{k}$.

2. $H_{n}^{p}=\left(\sum_{k=1}^{n} \frac{1}{k}\right)^{p} ; \quad H_{n}^{(p)}=\sum_{k=1}^{n} \frac{1}{k^{p}}$.

3. $h_{n}=\sum_{k=1}^{n} \frac{1}{2 k-1}=H_{2 n}-\frac{1}{2} H_{n} ; \quad h_{n}^{\prime}=\sum_{k=1}^{n} \frac{(-1)^{k+1}}{2 k-1}$.

$H_{n}^{(p)}$, the generalized harmonic number of order $p$, is sometime denoted by $\zeta_{n}(p)$ (Louis Comtet's Advanced Combinatorics, 1974, p. 217, [7b]). The alternating harmonic numbers, $H_{n}^{\prime}$, have been termed skew-harmonic numbers. We call $h_{n}$ the odd harmonic numbers and follow Berndt's notation ([17] p. 249, (8.2)) in preference to the symbol $O_{n}$ used by Borweins [8].

Taking the log of the two sides and then differentiating this product representation

$$
\frac{1}{\Gamma(x)}=x e^{\gamma x} \prod_{n=1}^{\infty}\left(1+\frac{x}{n}\right) e^{-x / n}
$$

yields

$$
\psi(x)=-\gamma-\frac{1}{x}+\sum_{n=1}^{\infty}\left(\frac{1}{n}-\frac{1}{x+n}\right)=-\gamma+\sum_{n=1}^{\infty}\left(\frac{1}{n}-\frac{1}{x+n-1}\right),
$$

leading to the telescoping sum:

$$
\psi(1+x)-\psi(x)=\sum_{n=1}^{\infty}\left(\frac{1}{x+n-1}-\frac{1}{x+n}\right)=\frac{1}{x}
$$

and for integral arguments $n \geq 1, \psi(n+1)-\psi(1)=H_{n}$ with $\psi(1)=-\gamma$.

We also have:

$$
h_{n}=\frac{1}{2}\left[\psi\left(n+\frac{1}{2}\right)-\psi\left(\frac{1}{2}\right)\right] .
$$

Choi ([18] Equation (3.7)) defined the harmonic numbers in terms of log-sine functions

$$
\begin{aligned}
H_{n} & =-4 n \int_{0}^{\frac{\pi}{2}} \log (\sin x) \sin x(\cos x)^{2 n-1} d x \\
& =-4 n \int_{0}^{\frac{\pi}{2}} \log (\cos x) \cos x(\sin x)^{2 n-1} d x
\end{aligned}
$$

An unusual, but intriguing representation has recently been given by Ciaurri et al. ([19] Equation (10)) as follows:

$$
H_{n}=\pi \int_{0}^{1}\left(x-\frac{1}{2}\right)\left(\frac{\cos \left(\frac{(4 n+1) \pi x}{2}\right)-\cos \left(\frac{\pi x}{2}\right)}{\sin \left(\frac{\pi x}{2}\right)}\right) d x
$$

We may introduce here the Lerch transcendent,

$$
\Phi(z, t, a)=\sum_{m=0}^{\infty} \frac{z^{m}}{(m+a)^{t}}
$$


which is defined for $|z|<1$ and $\Re(a)>0$ and satisfies the recurrence

$$
\Phi(z, t, a)=z \Phi(z, t, a+1)+a^{-t} .
$$

The Lerch transcendent generalizes the Hurwitz zeta function at $z=1$,

$$
\Phi(1, t, a)=\sum_{m=0}^{\infty} \frac{1}{(m+a)^{t}}
$$

and the polylogarithm, (see [14]), or de-Jonquière's function, when $a=1$,

$$
\operatorname{Li}_{t}(z):=\sum_{m=1}^{\infty} \frac{z^{m}}{m^{t}}, t \in \mathbb{C} \text { when }|z|<1 ; \Re(t)>1 \text { when }|z|=1 .
$$

The polylogarithm of negative integer order arises in the sums of the form

$$
\sum_{j \geq 1} j^{n} z^{j}=\operatorname{Li}_{-n}(z)=\frac{1}{(1-z)^{n+1}} \sum_{i=0}^{n-1}\left\langle\begin{array}{c}
n \\
i
\end{array}\right) z^{n-i},
$$

where the Eulerian number $\left\langle\begin{array}{c}n \\ i\end{array}\right\rangle=\sum_{j=0}^{i+1}(-1)^{j}\left(\begin{array}{c}n+1 \\ j\end{array}\right)(i-j+1)^{n}$, see [20]. The polygamma function of order $k$ for $z \neq-1,-2, \ldots$

$$
\psi^{(k)}(z)=\frac{d^{k}}{d z^{k}}\{\psi(z)\}=(-1)^{k+1} k ! \sum_{r=0}^{\infty} \frac{1}{(r+z)^{k+1}}
$$

and has the recurrence

$$
\psi^{(k)}(z+1)=\psi^{(k)}(z)+\frac{(-1)^{k} k !}{z^{k+1}} .
$$

The connection between the polygamma function and harmonic numbers is given by

$$
H_{z}^{(\alpha+1)}=\zeta(\alpha+1)+\frac{(-1)^{\alpha}}{\alpha !} \psi^{(\alpha)}(z+1), z \neq-1,-2,-3, \ldots
$$

and the multiplication formula for a positive integer $m$ is (Abramowitz and Stegun, Handbook of Mathematical Functions, p. 260, 6.4.8)

$$
\psi^{(k)}(m z)=\delta_{k, 0} \log (m)+\frac{1}{m^{k+1}} \sum_{j=0}^{m-1} \psi^{(k)}\left(z+\frac{j}{m}\right),
$$

where $\delta_{k, r}$ is the Kronecker delta.

We define the alternating zeta function (or Dirichlet eta function) $\eta(z)$ as

$$
\eta(z):=\sum_{n=1}^{\infty} \frac{(-1)^{n+1}}{n^{z}}=\left(1-2^{1-z}\right) \zeta(z)
$$

where $\eta(1)=\log 2$. If we put

$$
S(p, q):=\sum_{n=1}^{\infty} \frac{(-1)^{n+1} H_{n}^{(p)}}{n^{q}}
$$


in the case where $p$ and $q$ are both positive integers and weight $(p+q)$ is an odd integer, Flajolet and Salvy [15] gave the identity:

$$
\begin{aligned}
2 S(p, q) & =\left\{1-(-1)^{p}\right\} \zeta(p) \eta(q)+\eta(p+q) \\
& +2(-1)^{p} \sum_{i+2 k=q}\left(\begin{array}{c}
p+i-1 \\
p-1
\end{array}\right) \zeta(p+i) \eta(2 k) \\
& -2 \sum_{j+2 k=p}\left(\begin{array}{c}
q+j-1 \\
q-1
\end{array}\right)(-1)^{j} \eta(q+j) \eta(2 k),
\end{aligned}
$$

where $\eta(0)=\frac{1}{2}, \eta(1)=\log 2, \zeta(1)=0$, and $\zeta(0)=-\frac{1}{2}$ in accordance with the analytic continuation of the Riemann zeta function. For odd weight $(p+q)$, we have from [9] and ([15] Th.3.1):

$$
\begin{aligned}
& B W(p, q)=\sum_{n=1}^{\infty} \frac{H_{n}^{(p)}}{n^{q}}=(-1)^{p} \sum_{j=1}^{\left[\frac{q}{2}\right]}\left(\begin{array}{c}
p+q-2 j-1 \\
p-1
\end{array}\right) \zeta(p+q-2 j) \zeta(2 j) \\
& +\frac{1-(-1)^{p}}{2} \zeta(p) \zeta(q)+(-1)^{p} \sum_{j=1}^{\left[\frac{p}{2}\right]}\left(\begin{array}{c}
p+q-2 j-1 \\
q-1
\end{array}\right) \zeta(p+q-2 j) \zeta(2 j) \\
& +\frac{(-1)^{p}}{2}\left\{(-1)^{p}-\left(\begin{array}{c}
p+q-1 \\
p
\end{array}\right)-\left(\begin{array}{c}
p+q-1 \\
q
\end{array}\right)\right\} \zeta(p+q),
\end{aligned}
$$

where $[x]$ is the largest integer contained in $x$. It appears that some isolated cases of $B W(p, q)$, for even weight $(p+q)$, can be expressed in zeta terms, but, in general, almost certainly, for even weight $(p+q)$, and $p \geq 2$, no general closed form expression exists for $B W(p, q)$. Some examples with even weight are

$$
\sum_{n=1}^{\infty} \frac{H_{n}^{(2)}}{n^{4}}=\zeta^{2}(3)-\frac{1}{3} \zeta(6), \sum_{n=1}^{\infty} \frac{H_{n}^{(4)}}{n^{4}}=\frac{13}{12} \zeta(8) .
$$

There are also the two general forms of identities,

$$
2 \sum_{n=1}^{\infty} \frac{H_{n}^{(2 p+1)}}{n^{2 p+1}}=\zeta^{2}(2 p+1)+\zeta(4 p+2),
$$

and

$$
2 \sum_{n=1}^{\infty} \frac{H_{n}^{(2 p)}}{n^{2 p}}=\left(1-\frac{(4 p) ! B_{2 p}^{2}}{2((2 p) !)^{2} B_{4 p}}\right) \zeta(4 p)
$$

for $p \in \mathbb{N}$ and where $B_{p}$ are the signed Bernoulli numbers of the first kind.

Sofo [21], furthermore, developed the half integer Euler sums. For positive integers $m, p$ and odd weight $m+p$,

$$
\begin{aligned}
& W(m, p)=\sum_{n=1}^{\infty} \frac{H_{\frac{n}{2}}^{(m)}}{n^{p}}=(-1)^{p} \sum_{r=1}^{m} 2^{m-1}\left(\begin{array}{c}
m+p-1-r \\
m-r
\end{array}\right) \times \\
& {[B W(r, m+p-r)-S(r, m+p-r)]} \\
& +(-1)^{p+1} \sum_{r=2}^{m} \frac{1}{2^{p-r}}\left(\begin{array}{c}
m+p-1-r \\
m-r
\end{array}\right) \zeta(r) \zeta(m+p-r) \\
& +(-1)^{p+1} \sum_{k=2}^{p-1} \frac{(-1)^{k}}{2^{p-k}}\left(\begin{array}{c}
m+p-1-k \\
p-k
\end{array}\right) \zeta(k) \zeta(m+p-k) .
\end{aligned}
$$


In addition,

$$
\sum_{n \geq 1} \frac{H_{n}^{(m)}}{n^{p}}=2^{p-1} \sum_{n=1}^{\infty} \frac{H_{\frac{n}{2}}^{(m)}}{n^{p}}\left\{1-(-1)^{n+1}\right\} .
$$

We obtain the alternating Euler identity at half integer value,

$$
\sum_{n \geq 1} \frac{(-1)^{n+1} H_{\frac{n}{2}}^{(m)}}{n^{p}}=W(m, p)-2^{1-p} B W(m, p) .
$$

Some other Euler identities are given in [22-37].

\subsection{Cauchy Product and Relevant Generating Functions}

The multiplication of series by Cauchy's rule (Ch.VI of A. L. Cauchy's Cours d'Analyse de l'École Royale Polytechnique, 1821) is given by:

$$
\left(\sum_{k=0}^{\infty} a_{k} x^{k}\right)\left(\sum_{k=0}^{\infty} b_{k} x^{k}\right)=\sum_{k=0}^{\infty} c_{k} x^{k}
$$

with $c_{k}=\sum_{j=0}^{k} a_{j} b_{k-j}$. Cauchy showed (pp. 147-8, Th.6) that if the two convergent series on LHS are composed only of positive terms, i.e., they are absolutely convergent, then so is their product. Abel's theorem (1826) states that if all three series (including conditionally convergent) converge to sums, say $s_{a}, s_{b}, s_{c}$ respectively, then $s_{a} \cdot s_{b}=s_{c}$ necessarily.

A function $G(x)$ represented by the power series: $G(x):=\sum_{n=0}^{\infty} a_{n} x^{n}$ is known as ordinary generating function for the sequence $\left\{a_{n}\right\}$.

The Cauchy product of $(1-x)^{-1}$ and $\log (1-x)$ yields the generating function ([38] p. 54, 1.514.6):

$$
-\frac{\log (1-x)}{1-x}=\sum_{n=1}^{\infty} H_{n} x^{n}
$$

while the Cauchy product of $(1-x)^{-1}$ and $\log (1+x)$ yields:

$$
\frac{\log (1+x)}{1-x}=\sum_{n=1}^{\infty} H_{n}^{\prime} x^{n}
$$

both series converging for $|x|<1$.

\subsection{Few Sums for Further Use}

The Cauchy product of the numerical series $\sum_{k=0}^{\infty} \frac{(-1)^{k+1}}{k+1}$ with itself gives:

$$
2 \sum_{n=1}^{\infty} \frac{(-1)^{n+1}}{n+1}\left(1+\frac{1}{2}+\cdots+\frac{1}{n}\right)=2 \sum_{n=1}^{\infty}(-1)^{n+1} \frac{H_{n+1}}{n+1}-2 \sum_{n=1}^{\infty} \frac{(-1)^{n+1}}{(n+1)^{2}}
$$

Furthermore, if $u_{1}, u_{2}, u_{3}, \ldots$ is a decreasing sequence of positive numbers with limit 0 , then the series $u_{1}-\frac{1}{2}\left(u_{1}+u_{2}\right)+\frac{1}{3}\left(u_{1}+u_{2}+u_{3}\right)-\ldots$ is convergent. Hence, the series on LHS converges (with sum $\left.=2(-\log 2)^{2}\right)$ and so does the first series on RHS because the second series too is convergent with sum $=-2\left(1-\frac{\pi^{2}}{12}\right)$. We thus get:

$$
\sum_{n=1}^{\infty}(-1)^{n+1} \frac{H_{n}}{n+1}=\frac{\log ^{2}(2)}{2}
$$


From this, we deduce by a shift of the index:

$$
\sum_{n=1}^{\infty}(-1)^{n+1} \frac{H_{n}}{n}=\frac{\pi^{2}}{12}-\frac{\log ^{2}(2)}{2}
$$

leading to

$$
\sum_{n=1}^{\infty}(-1)^{n+1} \frac{H_{n}}{n(n+1)}=\frac{\pi^{2}}{12}-\log ^{2}(2)
$$

The Cauchy product of $\arctan x=\sum_{n=0}^{\infty} \frac{(-1)^{n}}{2 n+1} x^{n}$ with itself is the series ([39] p. 162, Ex. 13):

$$
(\arctan x)^{2}=\sum_{n=1}^{\infty}(-1)^{n+1} \frac{h_{n}}{n} x^{2 n} .
$$

The series are absolutely convergent when $|x|<1$ and convergent when $x=1$. ([40] p. 81, Ex. 2) Therefore,

$$
\sum_{n=1}^{\infty}(-1)^{n+1} \frac{h_{n}}{n}=\frac{\pi^{2}}{16}
$$

It occurs in ([41] pp. 322-323).

The software WolframAlpha gives:

$$
\int_{0}^{1}(\arctan x)^{2} d x=\frac{\pi}{16}(\pi+4 \log 2)-G
$$

where $G$ is Catalan's constant defined by $G=\sum_{n=1}^{\infty} \frac{(-1)^{n-1}}{(2 n-1)^{2}}=0.9159655 \ldots$

Thus, we have:

$$
\sum_{n=1}^{\infty}(-1)^{n+1} \frac{h_{n}}{n(2 n+1)}=\sum_{n=1}^{\infty}(-1)^{n+1}\left[\frac{h_{n}}{n}-\frac{2 h_{n}}{2 n+1}\right]=\frac{\pi^{2}}{16}+\frac{\pi \log 2}{4}-G .
$$

Putting the value from Equation (14) in Equation (15) leads to:

$$
\sum_{n=1}^{\infty}(-1)^{n+1} \frac{h_{n}}{2 n+1}=\frac{G}{2}-\frac{\pi \log 2}{8} .
$$

WolframAlpha gives:

$$
\int_{0}^{1} \frac{(\arctan x)^{2}}{x} d x=\frac{1}{8}[4 \pi G-7 \zeta(3)]
$$

yielding ([4] p.14, (4.16)):

$$
\sum_{n=1}^{\infty}(-1)^{n+1} \frac{h_{n}}{n^{2}}=\pi G-\frac{7 \zeta(3)}{4}
$$

\section{Euler-Like Alternating Sums}

\subsection{A New Sum}

It is easy to see that

$$
H_{2 n}^{2}=\left(\frac{1}{2} H_{n}+h_{n}\right)^{2}=\frac{1}{4} H_{n}^{2}+H_{n} h_{n}+h_{n}^{2}
$$


Therefore,

$$
\sum_{n=1}^{\infty}(-1)^{n+1} \frac{H_{2 n}^{2}}{n}=\sum_{n=1}^{\infty} \frac{(-1)^{n+1} H_{n}^{2}}{4 n}+\sum_{n=1}^{\infty} \frac{(-1)^{n+1} H_{n} h_{n}}{n}+\sum_{n=1}^{\infty} \frac{(-1)^{n+1} h_{n}^{2}}{n} .
$$

We discovered this sum independently:

$$
\sum_{n=1}^{\infty} \frac{(-1)^{n+1} H_{n}^{2}}{n}=\frac{3 \zeta(3)}{4}-\frac{\pi^{2} \log 2}{12}+\frac{\log ^{3}(2)}{3},
$$

which on shifting the index yields

$$
\sum_{n=1}^{\infty} \frac{(-1)^{n+1} H_{n}^{2}}{n+1}=-\frac{\zeta(3)}{4}+\frac{\pi^{2} \log 2}{12}-\frac{\log ^{3}(2)}{3}
$$

so that

$$
\sum_{n=1}^{\infty} \frac{(-1)^{n+1} H_{n}^{2}}{n(n+1)}=\zeta(3)-\frac{\pi^{2} \log 2}{6}+\frac{2 \log ^{3}(2)}{3},
$$

and we have an identity for Apery's constant,

$$
\sum_{n=1}^{\infty} \frac{(-1)^{n+1}(2 n+1) H_{n}^{2}}{n(n+1)}=\frac{\zeta(3)}{2} .
$$

Later on, we found the first two formulas recorded in ([12] Equations (4.4b) and (4.4c)), borrowed from De Doelder ([6] Equation (12)) who recorded the first sum with a typographical error.

We may also record here a related sum

$$
\sum_{n=1}^{\infty} \frac{(-1)^{n+1} H_{n}^{(2)}}{n}=\zeta(3)-\frac{\pi^{2} \log 2}{12}
$$

obtained via the formula of Flajolet and Salvy noted above.

We find this sum in ([6], Equation (21)):

$$
\sum_{n=1}^{\infty} \frac{(-1)^{n+1} h_{n}^{2}}{n}=\frac{7 \zeta(3)}{16} .
$$

This sum occurs in ([12], Equation (4.3b)):

$$
\sum_{n=1}^{\infty}(-1)^{n+1} \frac{H_{n-1}+H_{n}}{n} h_{n}=\frac{7 \zeta(3)}{8}-\frac{\pi^{2} \log 2}{8},
$$

that is,

$$
\sum_{n=1}^{\infty}(-1)^{n+1} \frac{2 H_{n} h_{n}}{n}-\sum_{n=1}^{\infty}(-1)^{n+1} \frac{h_{n}}{n^{2}}=\frac{7 \zeta(3)}{8}-\frac{\pi^{2} \log 2}{8} .
$$

We thus obtain using Equation (17):

$$
\sum_{n=1}^{\infty} \frac{(-1)^{n+1} H_{n} h_{n}}{n}=-\frac{7 \zeta(3)}{16}-\frac{\pi^{2} \log 2}{16}+\frac{\pi G}{2} .
$$

Using the values from Equations (19)-(22) in Equation (18), we deduce this new sum:

$$
\sum_{n=1}^{\infty}(-1)^{n+1} \frac{H_{2 n}^{2}}{2 n}=\frac{3 \zeta(3)}{32}-\frac{\pi^{2} \log 2}{24}+\frac{\log ^{3}(2)}{24}+\frac{\pi G}{4} .
$$


2.2. Double Sums for Catalan's Constant

We discovered this nice sum:

$$
\begin{aligned}
(1) 1-\left(1-\frac{1}{3}\right) \frac{1}{2}+ & \left(1-\frac{1}{3}+\frac{1}{5}\right) \frac{1}{3}-\cdots=\sum_{n=1}^{\infty}(-1)^{n+1} \frac{h_{n}^{\prime}}{n}=G . \\
\sum_{n=1}^{\infty}(-1)^{n+1} \frac{h_{n}^{\prime}}{n} & =\sum_{n=1}^{\infty}(-1)^{n+1} \frac{1}{n} \int_{0}^{1} \frac{1-(-1)^{n} x^{2 n}}{1+x^{2}} d x \\
& =\sum_{n=1}^{\infty}(-1)^{n+1} \frac{1}{n} \int_{0}^{1} \frac{1}{1+x^{2}} d x \\
& -\int_{0}^{1}\left(\sum_{n=1}^{\infty}(-1)^{2 n+1} \frac{x^{2 n}}{n}\right) \frac{1}{1+x^{2}} d x \\
& =\log (2) \frac{\pi}{4}-\int_{0}^{1} \frac{\log \left(1-x^{2}\right)}{1+x^{2}} d x \\
& =\frac{\pi}{4} \log 2-\left[\frac{\pi}{4} \log (2)-G\right]=G
\end{aligned}
$$

as

$$
\int_{0}^{1} \frac{\log \left(1-x^{2}\right)}{1+x^{2}} d x=\int_{0}^{1} \frac{\log (1+x)}{1+x^{2}} d x+\int_{0}^{1} \frac{\log (1-x)}{1+x^{2}} d x
$$

and ([38] p. 556, (4.291.8), (4.291.10)):

$$
\int_{0}^{1} \frac{\log (1+x)}{1+x^{2}} d x=\frac{\pi}{8} \log 2 ; \quad \int_{0}^{1} \frac{\log (1-x)}{1+x^{2}} d x=\frac{\pi}{8} \log 2-G
$$

We also found this sum by shifting the index in Equation (16):

$$
\sum_{n=1}^{\infty}(-1)^{n+1} \frac{h_{n}}{2 n-1}=\frac{G}{2}+\frac{\pi}{8} \log 2,
$$

for which Chu derives a wrong value $\frac{\pi^{2}}{24}+\frac{\pi \log (2)}{8}$ in ([12] Equation (4.5c)).

The combination of Equations (16) and (25) yields:

$$
\sum_{n=1}^{\infty} \frac{(-1)^{n+1} h_{n}}{4 n^{2}-1}=\frac{\pi \log 2}{8}
$$

and a new double sum for Catalan's constant:

$$
\sum_{n=1}^{\infty} \frac{(-1)^{n+1} n h_{n}}{4 n^{2}-1}=\frac{G}{4}
$$

We discovered this related sum:

$$
\sum_{n=1}^{\infty}(-1)^{n+1} \frac{h_{n}^{\prime}}{2 n-1}=\frac{3 \pi^{2}}{32}
$$




$$
\begin{aligned}
\sum_{n=1}^{\infty}(-1)^{n+1} \frac{h_{n}^{\prime}}{2 n-1} & =\sum_{n=1}^{\infty}(-1)^{n+1} \frac{1}{2 n-1} \int_{0}^{1} \frac{1-(-1)^{n} x^{2 n}}{1+x^{2}} d x \\
& =\sum_{n=1}^{\infty}(-1)^{n+1} \frac{1}{2 n-1} \int_{0}^{1} \frac{1}{1+x^{2}} d x \\
& -\int_{0}^{1}\left(\sum_{n=1}^{\infty}(-1)^{2 n+1} \frac{x^{2 n}}{2 n-1}\right) \frac{1}{1+x^{2}} d x \\
& =\left(\frac{\pi}{4}\right)^{2}+\int_{0}^{1} \frac{x \arctan h(x)}{1+x^{2}} d x \\
& =\frac{\pi^{2}}{16}+\frac{\pi^{2}}{32}=\frac{3 \pi^{2}}{32}
\end{aligned}
$$

\section{More Alternating Sums}

\subsection{Miscellaneous Double Sums}

We derived these sums earlier:

$$
\sum_{n=1}^{\infty}(-1)^{n+1} \frac{H_{n}}{n+1}=\frac{\log ^{2} 2}{2} ; \quad \sum_{n=1}^{\infty}(-1)^{n+1} \frac{H_{n}}{n}=\frac{\pi^{2}}{12}-\frac{\log ^{2}(2)}{2} .
$$

Furthermore,

$$
\sum_{n=1}^{\infty}(-1)^{n+1} \frac{H_{n}}{(n+1)^{2}}=\frac{\zeta(3)}{8} ; \quad \sum_{n=1}^{\infty}(-1)^{n+1} \frac{H_{n}}{n^{2}}=\frac{5 \zeta(3)}{8} .
$$

Since $H_{2 n}=h_{n}+\frac{H_{n}}{2}$, we deduce:

$$
\sum_{n=1}^{\infty}(-1)^{n+1} \frac{h_{n}}{n+1}=\frac{\pi}{16}(8-\pi)-\log 2 ; \quad \sum_{n=1}^{\infty}(-1)^{n+1} \frac{h_{n}}{n}=\frac{\pi^{2}}{16}
$$

and

$$
\sum_{n=1}^{\infty}(-1)^{n+1} \frac{h_{n}}{(n+1)^{2}}=\frac{7 \zeta(3)}{4}-\frac{\pi^{2}}{12}-\pi G+\pi-2 \log 2
$$

We also have these:

$$
\begin{gathered}
\sum_{n=1}^{\infty}(-1)^{n+1} \frac{H_{2 n}}{n^{3}}=-5 \operatorname{Li}_{4}\left(\frac{1}{2}\right)+\frac{13 \pi^{4}}{192}-\frac{35 \zeta(3) \log 2}{8}+\frac{5 \pi^{2} \log ^{2}(2)}{24}-\frac{5 \log ^{4}(2)}{24} \\
\sum_{n=1}^{\infty}(-1)^{n+1} \frac{H_{n}}{n^{3}}=-2 \operatorname{Li}_{4}\left(\frac{1}{2}\right)+\frac{11 \pi^{4}}{360}-\frac{7 \zeta(3) \log 2}{4}+\frac{\pi^{2} \log ^{2}(2)}{12}-\frac{\log ^{4}(2)}{12}
\end{gathered}
$$

Its terms (except the one with $\pi^{4}$ ) are $2 / 5$ times of the previous one. See the next sum with the same terms (except the one with $\pi^{4}$ ):

$$
\sum_{n=1}^{\infty}(-1)^{n+1} \frac{H_{n}^{2}}{n^{2}}=-2 \operatorname{Li}_{4}\left(\frac{1}{2}\right)+\frac{41 \pi^{4}}{1440}-\frac{7 \zeta(3) \log 2}{4}+\frac{\pi^{2} \log ^{2}(2)}{12}-\frac{\log ^{4}(2)}{4} .
$$

This sum is simpler:

$$
\sum_{n=1}^{\infty}(-1)^{n} \frac{H_{n}^{(3)}}{n}=\int_{0}^{1} \frac{\mathrm{Li}_{3}(-x)}{x(1+x)} d x=-\frac{19 \pi^{4}}{1440}+\frac{3 \zeta(3) \log 2}{4}
$$




$$
\begin{gathered}
\sum_{n=1}^{\infty}(-1)^{n+1} \frac{h_{n}}{n^{3}}=-4 \operatorname{Li}_{4}\left(\frac{1}{2}\right)+\frac{151 \pi^{4}}{2880}-\frac{7 \zeta(3) \log 2}{2}+\frac{\pi^{2} \log ^{2}(2)}{6}-\frac{\log ^{4}(2)}{6}, \\
\sum_{n=1}^{\infty}(-1)^{n+1} \frac{H_{2 n}}{(n+1)^{3}}=5 \operatorname{Li}_{4}\left(\frac{1}{2}\right)-\frac{181 \pi^{4}}{2880}+\frac{35 \zeta(3) \log 2}{8}-\frac{5 \pi^{2} \log ^{2}(2)}{24} \\
+\frac{5 \log ^{4} 2}{24}-\frac{3 \zeta(3)}{4}-\frac{\pi^{2}}{6}+2 \pi-4 \log 2 \\
\sum_{n=1}^{\infty}(-1)^{n+1} \frac{H_{n}}{(n+1)^{3}}=2 \operatorname{Li}_{4}\left(\frac{1}{2}\right)-\frac{15 \pi^{4}}{720}+\frac{7 \zeta(3) \log 2}{4}-\frac{\pi^{2} \log ^{2}(2)}{12}+\frac{\log ^{4}(2)}{12} \\
\sum_{n=1}^{\infty}(-1)^{n+1} \frac{h_{n}}{(n+1)^{3}}=4 \operatorname{Li}_{4}\left(\frac{1}{2}\right)-\frac{151 \pi^{4}}{2880}+\frac{7 \zeta(3) \log 2}{2}-\frac{\pi^{2} \log ^{2}(2)}{6} \\
+\frac{\log ^{4}(2)}{6}-\frac{3 \zeta(3)}{4}-\frac{\pi^{2}}{6}+2 \pi-4 \log 2 .
\end{gathered}
$$

3.2. Sums Involving Harmonic Numbers with Multiple Arguments

For multiple arguments, we know that, for $p \in \mathbb{N}, h_{p n}=H_{2 p n}-\frac{1}{2} H_{p n}$. From [42], we obtain

$$
\begin{gathered}
\sum_{n \geq 1} \frac{(-1)^{n+1} H_{p n}}{n}=\frac{1+p^{2}}{4 p} \zeta(2)-\frac{1}{2} \sum_{j=0}^{p-1} \log ^{2}\left(2 \sin \left(\frac{(2 j+1) \pi}{2 p}\right)\right), \\
\sum_{n \geq 1} \frac{(-1)^{n+1} H_{p n}}{n(n+1)}=\sum_{n \geq 1} \frac{(-1)^{n+1} H_{p n}}{n}-\sum_{n \geq 1} \frac{(-1)^{n+1} H_{p n}}{n+1}=\frac{p}{2} \zeta(2) \\
+H_{p-1} \ln 2-\sum_{j=0}^{p-1} \log ^{2}\left(2 \sin \left(\frac{(2 j+1) \pi}{2 p}\right)\right)-\frac{1}{2} \sum_{j=1}^{p-1} \frac{1}{j}\left(H_{-\frac{j}{2 p}}-H_{-\left(\frac{p+j}{2 p}\right)}\right),
\end{gathered}
$$

from which we ascertain

$$
\begin{gathered}
\sum_{n \geq 1} \frac{(-1)^{n+1} h_{p n}}{n(n+1)}=\frac{3 p}{4} \zeta(2)+\frac{1}{2}\left(H_{2 p-1}-H_{p-1}\right) \log 2 \\
+\frac{1}{2} \sum_{j=0}^{p-1} \log ^{2}\left(2 \sin \left(\frac{(2 j+1) \pi}{2 p}\right)\right)-\sum_{j=0}^{2 p-1} \log ^{2}\left(2 \sin \left(\frac{(2 j+1) \pi}{4 p}\right)\right) \\
+\frac{1}{4} \sum_{j=1}^{p-1} \frac{1}{j}\left(H_{-\frac{j}{2 p}}-H_{-\left(\frac{p+j}{2 p}\right)}\right)-\frac{1}{2} \sum_{j=1}^{2 p-1} \frac{1}{j}\left(H_{-\frac{j}{4 p}}-H_{-\left(\frac{p+j}{4 p}\right)}\right) .
\end{gathered}
$$

For the non-alternating case, we have, from [43],

$$
\sum_{n \geq 1} \frac{H_{p n}}{n(n+1)}=\frac{1}{p} \zeta(2)-\sum_{j=1}^{p-1} \frac{1}{j} H_{-\frac{j}{p}}
$$

and, therefore, after some simplification

$$
\sum_{n \geq 1} \frac{h_{p n}}{n(n+1)}=\frac{1}{2} \sum_{j=1}^{p-1} \frac{1}{j} H_{-\frac{j}{p}}-\sum_{j=1}^{2 p-1} \frac{1}{j} H_{-\frac{j}{2 p}}=-\sum_{j=1}^{p} \frac{1}{(2 j-1)} H_{-\frac{(2 j-1)}{2 p}} .
$$


A simple calculation gives us

$$
\sum_{n \geq 1} \frac{h_{2 n}}{n(2 n+1)}=\log (2)+\frac{1}{2} \pi-\frac{3}{4} \zeta(2) .
$$

3.3. Few Relations between Pairs of Sums

We discovered these relations:

$$
\sum_{n=1}^{\infty} \frac{(-1)^{n+1} h_{n}^{2}}{2 n-1}+\sum_{n=1}^{\infty} \frac{(-1)^{n+1} h_{n}}{(2 n+1)^{2}}=\frac{\pi^{3}}{48}+\frac{\pi \log ^{2}(2)}{16}
$$

and

$$
\sum_{n=1}^{\infty} \frac{(-1)^{n+1} h_{n}^{2}}{2 n+1}+\sum_{n=1}^{\infty} \frac{(-1)^{n+1} h_{n}}{(2 n+1)^{2}}=\frac{\pi^{3}}{96}-\frac{\pi \log ^{2}(2)}{16}
$$

Thus,

$$
\sum_{n=1}^{\infty} \frac{(-1)^{n+1} h_{n}^{2}}{2 n-1}-\sum_{n=1}^{\infty} \frac{(-1)^{n+1} h_{n}^{2}}{2 n+1}=\frac{\pi^{3}}{96}+\frac{\pi \log ^{2}(2)}{8}
$$

leading to the sum:

$$
\sum_{n=1}^{\infty} \frac{(-1)^{n+1} h_{n}^{2}}{4 n^{2}-1}=\frac{\pi^{3}}{192}+\frac{\pi \log ^{2}(2)}{16} .
$$

This relation was deduced from ([12] Equation (4.6c)):

$$
\sum_{n=1}^{\infty} \frac{(-1)^{n+1} h_{n}^{2}}{2 n-1}-\sum_{n=1}^{\infty} \frac{(-1)^{n+1} h_{n}}{(2 n-1)^{2}}=-\frac{\pi^{3}}{96}+\frac{\pi \log ^{2}(2)}{16} .
$$

We thus have:

$$
\begin{gathered}
\sum_{n=1}^{\infty} \frac{(-1)^{n+1} h_{n}^{2}}{2 n-1}+\sum_{n=1}^{\infty} \frac{(-1)^{n+1} h_{n}}{(2 n+1)^{2}}=\frac{\pi^{3}}{48}+\frac{\pi \log ^{2}(2)}{16}, \\
\sum_{n=1}^{\infty} \frac{(-1)^{n+1} h_{n}}{(2 n-1)^{2}}+\sum_{n=1}^{\infty} \frac{(-1)^{n+1} h_{n}}{(2 n+1)^{2}}=\frac{\pi^{3}}{32}=\sum_{n=1}^{\infty} \frac{(-1)^{n+1}}{(2 n-1)^{3}}, \\
\sum_{n=1}^{\infty}(-1)^{n+1} \frac{h_{n}}{n(n+1)}=\frac{\pi^{2}}{8}-\frac{\pi}{2}+\log 2, \\
\sum_{n=1}^{\infty}(-1)^{n+1} \frac{h_{n}}{n^{2}(n+1)^{2}}=-\frac{\pi^{2}}{3}+2 \pi-4 \log 2 .
\end{gathered}
$$

The two preceding sums have similar terms yielding a nice sum for $\zeta(2)$ :

$$
\begin{gathered}
\sum_{n=1}^{\infty}(-1)^{n+1}\left(\frac{2 n+1}{n(n+1)}\right)^{2} h_{n}=\frac{\pi^{2}}{6}, \\
\sum_{n=1}^{\infty}(-1)^{n+1} \frac{h_{n}}{n^{3}(n+1)^{3}}=\frac{151 \pi^{4}}{1440}-7 \zeta(3) \log 2+\frac{\pi^{2} \log ^{2}(2)}{3} \\
-\frac{\log ^{4} 2}{3}+\frac{3 \zeta(3)}{4}+\frac{7 \pi^{2}}{6}-8 \pi+16 \log 2-8 \operatorname{Li}_{4}\left(\frac{1}{2}\right) .
\end{gathered}
$$




\section{Integrals and Euler Sums}

In this section, we will explore the connection of integrals involving inverse trigonometric and hyperbolic functions with Euler sums, thereby producing a number of interesting and new identities.

Theorem 1. Let $a \in \mathbb{R}^{+} \cup\{0\}, \delta \in \mathbb{R}^{+} \backslash\{0\}, p \in \mathbb{N} \cup\{0\}$ and $q \in \mathbb{R}^{+}$; then,

$$
\begin{aligned}
I(a, \delta, p, q) & =\int_{0}^{1} x^{a}\left(\tanh ^{-1}\left(\delta x^{q}\right)\right)^{2} \log ^{p}(x) d x \\
& =(-1)^{p} p ! \sum_{n \geq 1} \frac{\delta^{2 n} h_{n}}{n(2 q n+a+1)^{p+1}},
\end{aligned}
$$

where

$$
h_{n}=H_{2 n}-\frac{1}{2} H_{n}=\log (2)+\frac{1}{2} H_{n-\frac{1}{2}}
$$

and $H_{n}, H_{2 n}$ are harmonic numbers with unitary argument and double argument.

Proof. We have that

$$
\left(\tanh ^{-1}\left(\delta x^{q}\right)\right)^{2}=\sum_{n \geq 1} \frac{\delta^{2 n} h_{n} x^{2 q n}}{n},
$$

therefore

$$
I(a, \delta, p, q)=\sum_{n \geq 1} \frac{\delta^{2 n} h_{n}}{n} \int_{0}^{1} x^{2 q n+a} \log ^{p}(x) d x,
$$

and, integrating by parts, yields

$$
I(a, \delta, p, q)=(-1)^{p} p ! \sum_{n \geq 1} \frac{\delta^{2 n} h_{n}}{n(2 q n+a+1)^{p+1}} .
$$

Certain particular interesting cases can be explicitly represented and we detail some cases in the next few corollaries.

Corollary 1. Let $a=-1, \delta= \pm 1, p \in \mathbb{N} \cup\{0\}$ and $q \in \mathbb{R}^{+}$; then,

$$
\begin{aligned}
I(-1, \pm 1, p, q) & =\int_{0}^{1} \frac{1}{x}\left(\tanh ^{-1}\left( \pm x^{q}\right)\right)^{2} \log ^{p}(x) d x \\
& =\frac{(-1)^{p} p !}{(2 q)^{p+1}} \sum_{n \geq 1} \frac{h_{n}}{n^{p+2}} .
\end{aligned}
$$

For $p=0$,

$$
\begin{aligned}
I(-1, \pm 1,0, q) & =\int_{0}^{1} \frac{1}{x}\left(\tanh ^{-1}\left( \pm x^{q}\right)\right)^{2} d x \\
& =\frac{1}{(2 q)^{1}} \sum_{n \geq 1} \frac{h_{n}}{n^{2}}=\frac{7}{8 q} \zeta(3) .
\end{aligned}
$$


For $p=1$,

$$
\begin{aligned}
I(-1, \pm 1,1, q) & =\int_{0}^{1} \frac{1}{x}\left(\tanh ^{-1}\left( \pm x^{q}\right)\right)^{2} \log (x) d x \\
& =-\frac{1}{(2 q)^{2}} \sum_{n \geq 1} \frac{h_{n}}{n^{3}}=\frac{1}{4 q^{2}}\left(4 L(3)-\frac{35}{8} \zeta(4)\right)
\end{aligned}
$$

where

$$
L(3)=\frac{11}{4} \zeta(4)-\frac{7}{4} \zeta(3) \log 2+\frac{1}{2} \zeta(2) \log ^{2}(2)-\frac{1}{12} \log ^{4}(2)-2 \operatorname{Li}_{4}\left(\frac{1}{2}\right) .
$$

For $p$ even, let $p=2(m-1), m \in \mathbb{N}$

$$
\begin{aligned}
I(-1, \pm 1,2(m-1), q) & =\int_{0}^{1} \frac{1}{x}\left(\tanh ^{-1}\left( \pm x^{q}\right)\right)^{2} \log ^{2 m-2}(x) d x \\
& =-\frac{(2 m-2) !}{(2 q)^{2 m+1}} \sum_{n \geq 1} \frac{h_{n}}{n^{2 m}}=\frac{(2 m-2) !}{(2 q)^{2 m+1}} Q(m),
\end{aligned}
$$

where, from paper [44],

$$
\begin{aligned}
Q(m)= & \sum_{n \geq 1} \frac{h_{n}}{n^{2 m}}=\frac{2^{2 m+1}-1}{4} \zeta(2 m+1) \\
& -\frac{1}{2} \sum_{j=1}^{m-1}\left(2^{2 m-2 j+1}-1\right) \zeta(2 j) \zeta(2 m-2 j+1) .
\end{aligned}
$$

For

$$
\begin{aligned}
& I\left(-1, \pm \frac{1}{3}, 0,1\right)=\int_{0}^{1} \frac{1}{x}\left(\tanh ^{-1}\left( \pm \frac{1}{3} x\right)\right)^{2} d x \\
&=\frac{1}{2} \sum_{n \geq 1} \frac{h_{n}}{3^{2 n} n^{2}} \\
&=\frac{1}{8} \mathrm{Li}_{3}\left(\frac{1}{4}\right)+\frac{1}{4} \mathrm{Li}_{2}\left(\frac{1}{4}\right) \log 2-\frac{1}{12} \log \left(\frac{27}{16}\right) \log ^{2}(2),
\end{aligned}
$$

where we have the identity

$$
\sum_{n \geq 1} \frac{H_{n}}{3^{2 n} n^{2}}=\mathrm{Li}_{3}\left(\frac{1}{9}\right)+\zeta(3)-\mathrm{Li}_{3}\left(\frac{8}{9}\right) \log (2)-\mathrm{Li}_{2}\left(\frac{8}{9}\right) \log \left(\frac{9}{8}\right)-\log ^{2}\left(\frac{9}{8}\right) \log 3
$$

Corollary 2. Let $a=2 q-1, \delta= \pm 1, p \in \mathbb{N} \cup\{0\}$ and $q \in \mathbb{R}^{+}$; then,

$$
\begin{aligned}
I(2 q-1, \pm 1, p, q) & =\int_{0}^{1} \frac{1}{x}\left(\tanh ^{-1}\left( \pm x^{q}\right)\right)^{2} \log ^{p}(x) d x \\
& =\frac{(-1)^{p} p !}{(2 q)^{p+1}} \sum_{n \geq 1} \frac{h_{n}}{n(n+1)^{p+1}} \\
& =\frac{(-1)^{p} p !}{(2 q)^{p+1}} \sum_{n \geq 1} h_{n}\left(\frac{1}{n(n+1)}-\sum_{j=2}^{p+1} \frac{1}{(n+1)^{j}}\right) .
\end{aligned}
$$


For $p=2, q=1$

$$
\begin{aligned}
I(1, \pm 1,2,1) & =\int_{0}^{1} x\left(\tanh ^{-1}( \pm x)\right)^{2} \log ^{2}(x) d x \\
& =\frac{1}{4} \sum_{n \geq 1} \frac{h_{n}}{n(n+1)^{3}} \\
& =\frac{1}{4} \sum_{n \geq 1} h_{n}\left(\frac{1}{n(n+1)}-\sum_{j=2}^{3} \frac{1}{(n+1)^{j}}\right) \\
& =L(3)+\frac{7}{2} \log (2)-\frac{3}{4} \zeta(2)-\frac{11}{16} \zeta(3)-\frac{35}{32} \zeta(4),
\end{aligned}
$$

where we have evaluated

$$
\begin{gathered}
\sum_{n \geq 1} \frac{h_{n}}{n(n+1)}=2 \log 2, \sum_{n \geq 1} \frac{h_{n}}{(n+1)^{2}}=\zeta(2)-4 \log (2)+\frac{7}{4} \zeta(3), \\
\sum_{n \geq 1} \frac{h_{n}}{(n+1)^{3}}=2 \zeta(2)-4 L(3)-8 \log 2+\zeta(3)+\frac{35}{8} \zeta(4) .
\end{gathered}
$$

For $\delta= \pm \frac{1}{3}$

$$
\begin{aligned}
I\left(1, \pm \frac{1}{3}, 0,1\right) & =\int_{0}^{1} x\left\{\tanh ^{-1}\left( \pm \frac{1}{3} x\right)\right\}^{2} d x=\frac{1}{2} \sum_{n \geq 1} \frac{h_{n}}{9^{n} n(n+1)} \\
& =15 \log 2-9 \log 3-\log ^{2}(2)
\end{aligned}
$$

where

$$
\sum_{n \geq 1} \frac{H_{n}}{9^{n} n(n+1)}=\mathrm{Li}_{2}\left(\frac{1}{9}\right)-4 \log ^{2}\left(\frac{9}{8}\right) .
$$

We can decompose the two sums into:

$$
\sum_{n=1}^{\infty} \frac{h_{n}}{9^{n} n}=\frac{1}{4} \log ^{2}(2) ; \quad \sum_{n=1}^{\infty} \frac{h_{n}}{9^{n+1}(n+1)}=\frac{1}{4} \log ^{2}(2)-\frac{10}{3} \log 2+2 \log 3,
$$

and

$$
\sum_{n=1}^{\infty} \frac{H_{n}}{9^{n} n}=\mathrm{Li}_{2}\left(\frac{1}{9}\right)+\frac{1}{2} \log ^{2}\left(\frac{9}{8}\right) ; \quad \sum_{n=1}^{\infty} \frac{H_{n}}{9^{n+1}(n+1)}=\frac{1}{2} \log ^{2}\left(\frac{9}{8}\right) .
$$

Moreover,

$$
\sum_{n=1}^{\infty} \frac{H_{2 n}}{9^{2 n+1}(2 n+1)}=\frac{1}{4}\left[\log ^{2}\left(\frac{9}{8}\right)-\log ^{2}\left(\frac{10}{9}\right)\right]=\frac{1}{4} \log \left(\frac{81}{80}\right) \log \left(\frac{5}{4}\right) .
$$

An interesting connection with these given four sums is the alternating identity

$$
\sum_{n=1}^{\infty} \frac{(-1)^{n+1} H_{n}}{3^{n}(n+1)}=\frac{3}{2} \log ^{2}\left(\frac{4}{3}\right)
$$

from which, by extrapolation, we are able to obtain the very fast converging alternating identity

$$
\zeta(2)=2 \sum_{n=1}^{\infty} \frac{(-1)^{n+1} h_{n}}{3^{n-1} n}
$$


Corollary 3. For $a=-1-q$

$$
\begin{aligned}
I(-q-1, \pm 1, p, q) & =\int_{0}^{1} x^{-1-q}\left(\tanh ^{-1}\left( \pm x^{q}\right)\right)^{2} \log ^{p}(x) d x \\
& =\frac{(-1)^{p} p !}{(2 q)^{p+1}} \sum_{n \geq 1} \frac{h_{n}}{n(2 n-1)^{p+1}} \\
& =\frac{(-1)^{p} p !}{q^{p+1}} \sum_{n \geq 1} h_{n}\left(\frac{(-1)^{p}}{n(2 n-1)}+2 \sum_{j=2}^{p+1} \frac{(-1)^{p+1+j}}{(2 n-1)^{j}}\right) \\
& =\frac{p !}{q^{p+1}}\left(\zeta(2)+2 \sum_{j=2}^{p+1}(-1)^{1+j} V(j)\right),
\end{aligned}
$$

where, from paper [44], we have evaluated, for $j$ even,

$$
\begin{aligned}
V(j)= & \sum_{n \geq 1} \frac{h_{n}}{(2 n-1)^{j}}=\frac{1}{4}(\zeta(j+1)+\eta(j+1))+\frac{1}{2}(\zeta(j)+\eta(j)) \log 2 \\
& -\frac{1}{2^{j+1}} \sum_{k=1}^{\frac{j}{2}-1}\left(2^{2 k}-1\right) \zeta(2 k) \zeta(j-2 k+1) .
\end{aligned}
$$

For the case

$$
\begin{aligned}
I(-2, \pm 1,2,1)= & \int_{0}^{1} x^{-2}\left(\tanh ^{-1}( \pm x)\right)^{2} \log ^{2}(x) d x=2 L(3)-\frac{5}{16} \zeta(4) \\
& +\frac{7}{2} \zeta(3) \log 2-\frac{7}{4} \zeta(3)-3 \zeta(2) \log 2+2 \zeta(2)
\end{aligned}
$$

where we have the results

$$
\begin{gathered}
\sum_{n \geq 1} \frac{h_{n}}{n(2 n-1)}=\zeta(2), \sum_{n \geq 1} \frac{h_{n}}{(2 n-1)^{2}}=\frac{3}{4} \zeta(2) \log 2+\frac{7}{16} \zeta(3), \\
\sum_{n \geq 1} \frac{h_{n}}{(2 n-1)^{3}}=\frac{21}{2} L(3)+\frac{7}{8} \zeta(3) \log 2-\frac{5}{64} \zeta(4) .
\end{gathered}
$$

For

$$
\begin{aligned}
I\left(-2, \pm \frac{1}{2}, 0,1\right) & =\int_{0}^{1} x^{-2}\left\{\tanh ^{-1}\left( \pm \frac{1}{2} x\right)\right\}^{2} d x=\sum_{n \geq 1} \frac{h_{n}}{2^{2 n} n(2 n-1)} \\
& =\frac{1}{2} \zeta(2)-\frac{1}{2} \operatorname{Li}_{2}\left(\frac{1}{3}\right)-\frac{1}{8} \log \left(\frac{243}{16}\right) \log 3
\end{aligned}
$$

where we have the identity

$$
\sum_{n \geq 1} \frac{H_{2 n}}{2^{2 n} n(2 n-1)}=\frac{5}{4} \zeta(2)+\frac{3}{2} \log \left(\frac{3}{2}\right)-\frac{1}{2} \log 2-\frac{3}{2} \operatorname{Li}_{2}\left(\frac{2}{3}\right)-\frac{3}{2} \log \left(\frac{3}{2}\right) \log 3 .
$$

Higher powers of the $\tanh ^{-1}(x)$ function yield more Euler sum identities, and we have the following corollary. 


\section{Corollary 4.}

$$
\begin{aligned}
\int_{0}^{1} x^{a}\left(\tanh ^{-1}(x)\right)^{3} d x & =\sum_{n \geq 1} \frac{h_{n}}{n} \sum_{j \geq 1} \frac{1}{(2 j-1)} \int_{0}^{1} x^{2 n+2 j+a-1} d x \\
& =\sum_{n \geq 1} \sum_{j \geq 1} \frac{h_{n}}{n(2 j-1)(2 n+2 j+a)} \\
& =\sum_{n \geq 1} \frac{h_{n}\left(2 \log (2)+H_{n+\frac{a}{2}}\right)}{n(2 n+a+1)} .
\end{aligned}
$$

Various cases follow, for $a=-1$,

$$
\int_{0}^{1} \frac{1}{x}\left(\tanh ^{-1}(x)\right)^{3} d x=\sum_{n \geq 1} \frac{h_{n}\left(2 \log (2)+H_{n-\frac{1}{2}}\right)}{4 n^{2}}=\sum_{n \geq 1} \frac{h_{n}^{2}}{4 n^{2}}=\frac{45}{32} \zeta(4) .
$$

For $a=1$,

$$
\begin{aligned}
& \int_{0}^{1} x\left(\tanh ^{-1}(x)\right)^{3} d x=\frac{1}{4} \sum_{n \geq 1} \frac{h_{n}\left(2 \log (2)+H_{n+\frac{1}{2}}\right)}{n(n+1)} \\
& =\frac{1}{4} \sum_{n \geq 1}\left(\frac{2 h_{n}}{n(n+1)(2 n+1)}+\frac{h_{n}^{2}}{n(n+1)}\right)=\frac{3}{4} \zeta(2) .
\end{aligned}
$$

From here, we have the identities

$$
\sum_{n \geq 1} \frac{h_{n}}{n(n+1)(2 n+1)}=\zeta(2)-2 \log 2, \sum_{n \geq 1} \frac{h_{n}^{2}}{n(n+1)}=\frac{1}{2} \zeta(2)+2 \log 2,
$$

and we have the new identities

$$
\begin{gathered}
\zeta(2)=\frac{2}{3} \sum_{n \geq 1} \frac{h_{n}}{n(n+1)}\left(h_{n}+\frac{1}{2 n+1}\right), \\
\sum_{n \geq 1} \frac{h_{n} H_{\frac{n}{2}}}{n(n+1)}=\frac{21}{16} \zeta(3)-\frac{9}{2} \log ^{2}(2)+2 G+\frac{1}{2} \zeta(2) .
\end{gathered}
$$

For $a=-2$,

$$
\begin{aligned}
& \int_{0}^{1} \frac{1}{x^{2}}\left(\tanh ^{-1}(x)\right)^{3} d x=\frac{1}{2} \sum_{n \geq 1} \frac{h_{n}\left(2 \log (2)+H_{n-1}\right)}{n(2 n-1)} \\
& =\sum_{n \geq 1}\left(\frac{h_{n} \log 2}{n(2 n-1)}+\frac{h_{n} H_{n}}{2 n(2 n-1)}-\frac{h_{n}}{2 n^{2}(2 n-1)}\right)=\frac{3}{2} \zeta(3) .
\end{aligned}
$$

From here, we have the identities

$$
\begin{gathered}
\sum_{n \geq 1} \frac{h_{n}}{n(2 n-1)}=\zeta(2), \sum_{n \geq 1} \frac{h_{n}}{n^{2}}=\frac{7}{4} \zeta(3), \\
\sum_{n \geq 1} \frac{h_{n} H_{n}}{n(2 n-1)}=\frac{5}{4} \zeta(3)-2 \zeta(2) \log 2+2 \zeta(2) .
\end{gathered}
$$


Consider the quartic,

$$
\begin{gathered}
\int_{0}^{1} \frac{1}{x}\left(\tanh ^{-1}(x)\right)^{4} d x=\frac{1}{2} \sum_{n \geq 1} \sum_{j \geq 1} \frac{h_{n} h_{j}}{n j(n+j)}=\frac{93}{32} \zeta(5) . \\
\int_{0}^{1} \frac{1}{\sqrt{x}}\left(\tanh ^{-1}\left(\frac{1}{2} x^{\frac{1}{4}}\right)\right)^{4} d x=\frac{1}{2} \sum_{n \geq 1} \sum_{j \geq 1} \frac{h_{n} h_{j}}{2^{2 n+2 j-1} n j(n+j+1)} \\
=36 \zeta(3)+22 \log ^{3}(3)-\frac{3}{8} \log ^{4}(3)-48 \log (2) \log ^{2}(3)+48 \log (3) \operatorname{Li}_{2}\left(-\frac{1}{3}\right)+48 \operatorname{Li}_{3}\left(-\frac{1}{3}\right) .
\end{gathered}
$$

For powers of the $\arctan x$ function, we shall find some further alternating harmonic Euler sums.

Theorem 2. Let $a \in \mathbb{R}^{+} \cup\{0\}, \delta \in \mathbb{R}^{+} \backslash\{0\}, p \in \mathbb{N} \cup\{0\}$ and $q \in \mathbb{R}^{+}$; then,

$$
\begin{aligned}
J(a, \delta, p, q) & =\int_{0}^{1} x^{a} \arctan ^{2}\left(\delta x^{q}\right) \log ^{p}(x) d x \\
& =(-1)^{p} p ! \sum_{n \geq 1} \frac{(-1)^{n+1} \delta^{2 n} h_{n}}{n(2 q n+a+1)^{p+1}},
\end{aligned}
$$

where

$$
h_{n}=H_{2 n}-\frac{1}{2} H_{n}=\log 2+\frac{1}{2} H_{n-\frac{1}{2}}
$$

and $\mathrm{H}_{n}, \mathrm{H}_{2 n}$ are harmonic numbers with unitary argument and double argument.

Proof. We have that

$$
\arctan ^{2}\left(\delta x^{q}\right)=\sum_{n \geq 1} \frac{(-1)^{n+1} \delta^{2 n} h_{n} x^{2 q n}}{n}
$$

therefore,

$$
\begin{aligned}
J(a, \delta, p, q) & =\sum_{n \geq 1} \frac{(-1)^{n+1} \delta^{2 n} h_{n}}{n} \int_{0}^{1} x^{2 q n+a} \log ^{p}(x) d x \\
& =(-1)^{p} p ! \sum_{n \geq 1} \frac{(-1)^{n+1} \delta^{2 n} h_{n}}{n(2 q n+a+1)^{p+1}} .
\end{aligned}
$$

Certain particular interesting cases can be explicitly represented and we detail some cases in the next few corollaries.

Corollary 5. Let $a=-1, \delta= \pm 1, p \in \mathbb{N} \cup\{0\}$ and $q \in \mathbb{R}^{+}$; then,

$$
\begin{aligned}
J(-1, \pm 1, p, q) & =\int_{0}^{1} \frac{1}{x} \arctan ^{2}\left( \pm x^{q}\right) \log ^{p}(x) d x \\
& =\frac{(-1)^{p} p !}{(2 q)^{p+1}} \sum_{n \geq 1} \frac{(-1)^{n+1} h_{n}}{n^{p+2}} .
\end{aligned}
$$


For $p=1$, and using the results of the previous sections,

$$
\begin{aligned}
& J(-1, \pm 1,1, q)=\int_{0}^{1} \frac{1}{x} \arctan ^{2}\left( \pm x^{q}\right) \log (x) d x=\frac{1}{(2 q)^{2}} \sum_{n \geq 1} \frac{(-1)^{n+1} h_{n}}{n^{3}} \\
= & \frac{1}{(2 q)^{2}}\left\{\frac{151}{32} \zeta(4)-\frac{7}{2} \zeta(3) \log 2+\zeta(2) \log ^{2}(2)-\frac{1}{6} \log ^{4}(2)-4 \operatorname{Li}_{4}\left(\frac{1}{2}\right)\right\} .
\end{aligned}
$$

Corollary 6. Let $a=2 q-1, \delta= \pm 1, p \in \mathbb{N} \cup\{0\}$ and $q \in \mathbb{R}^{+}$; then,

$$
\begin{aligned}
J(2 q-1, \pm 1, p, q) & =\int_{0}^{1} x^{2 q-1} \arctan ^{2}\left( \pm x^{q}\right) \log ^{p}(x) d x \\
& =\frac{(-1)^{p} p !}{(2 q)^{p+1}} \sum_{n \geq 1} \frac{(-1)^{n+1} h_{n}}{n(n+1)^{p+1}} . \\
& =\frac{(-1)^{p} p !}{(2 q)^{p+1}} \sum_{n \geq 1}(-1)^{n+1} h_{n}\left(\frac{1}{n}-\sum_{j=1}^{p+1} \frac{1}{(n+1)^{j}}\right) .
\end{aligned}
$$

For $p=2$, and using the results of the previous sections,

$$
\begin{aligned}
J(2 q-1, \pm 1,2, q) & =\int_{0}^{1} x^{2 q-1} \arctan ^{2}\left( \pm x^{q}\right) \log ^{2}(x) d x \\
& =\frac{2}{(2 q)^{p+1}} \sum_{n \geq 1}(-1)^{n+1} h_{n}\left(\frac{1}{n}-\sum_{j=1}^{3} \frac{1}{(n+1)^{j}}\right) \\
= & \frac{1}{(2 q)^{2}}\left(\begin{array}{c}
\pi G+\frac{151}{32} \zeta(4)-\frac{7}{2} \zeta(3) \log 2+\zeta(2) \log ^{2}(2)-\frac{1}{6} \log ^{4}(2) \\
-4 \operatorname{Li}_{4}\left(\frac{1}{2}\right)-\frac{3 \pi}{2}+\frac{9}{4} \zeta(2)+7 \log 2-\zeta(3)
\end{array}\right) .
\end{aligned}
$$

Corollary 7. Let $a=-q-1, \delta= \pm 1, p \in \mathbb{N} \cup\{0\}$ and $q \in \mathbb{R}^{+}$; then,

$$
\begin{aligned}
& J(-q-1, \pm 1, p, q)=\int_{0}^{1} x^{-q-1} \arctan ^{2}\left( \pm x^{q}\right) \log ^{p}(x) d x \\
&=\frac{(-1)^{p} p !}{q^{p+1}} \sum_{n \geq 1} \frac{(-1)^{n+1} h_{n}}{n(2 n-1)^{p+1}} . \\
&=\frac{(-1)^{p} p !}{q^{p+1}} \sum_{n \geq 1}(-1)^{n+1} h_{n}\left(\frac{(-1)^{p+1}}{n}+2 \sum_{j=1}^{p+1} \frac{(-1) j++^{p+1}}{(2 n-1)^{j}}\right) .
\end{aligned}
$$

For $p=0$,

$$
\begin{aligned}
J(2 q-1, \pm 1,0, q) & =\int_{0}^{1} x^{-q-1} \arctan ^{2}\left( \pm x^{q}\right) d x \\
& =\frac{1}{q} \sum_{n \geq 1}(-1)^{n+1} h_{n}\left(-\frac{1}{n}+\frac{2}{(2 n-1)}\right) \\
& =\frac{1}{q}\left(\frac{\pi}{4} \log 2+G-\frac{3}{8} \zeta(2)\right) .
\end{aligned}
$$


Higher powers of the $\arctan (x)$ function yield more Euler sum identities, and we have the following corollary.

\section{Corollary 8.}

$$
\begin{aligned}
\int_{0}^{1} x^{a} \arctan ^{3}(x) d x & =\sum_{n \geq 1} \frac{(-1)^{n+1} h_{n}}{n} \int_{0}^{1} x^{2 n+a} \arctan (x) d x \\
& =\sum_{n \geq 1} \frac{(-1)^{n+1} h_{n}}{4 n}\left(\frac{\pi+H_{\frac{2 n+a-2}{4}}-H_{\frac{2 n+a}{4}}}{(2 n+a+1)}\right) .
\end{aligned}
$$

Various cases follow, for $a=0$, and using the double argument of the polygamma function

$$
\begin{aligned}
\int_{0}^{1} \arctan ^{3}(x) d x & =\sum_{n \geq 1} \frac{(-1)^{n+1} h_{n}}{4 n}\left(\frac{\pi+H_{\frac{2 n-2}{4}}-H_{\frac{2 n}{4}}}{2 n+1}\right) \\
& =\sum_{n \geq 1} \frac{(-1)^{n+1} h_{n}}{4 n(2 n+1)}\left(\pi-2 \log (2)+2 H_{n}-2 H_{\frac{n}{2}}\right) \\
= & \frac{63}{64} \zeta(3)-\frac{3}{4} \pi G+\frac{\pi^{3}}{64}+\frac{18}{32} \zeta(2) \log 2 .
\end{aligned}
$$

Manipulating this integral identity gives us the new Euler sums,

$$
\begin{gathered}
\sum_{n \geq 1} \frac{(-1)^{n+1} h_{n} H_{\frac{n}{2}}}{n}=\pi G-\frac{35}{32} \zeta(3)-\frac{9}{8} \zeta(2) \log 2, \\
\sum_{n \geq 1} \frac{(-1)^{n+1} h_{n}\left(H_{\frac{n}{2}}-H_{n}\right)}{2 n+1}=\frac{21}{32} \zeta(3)-\frac{1}{4} \pi G+\frac{3}{4} \pi \log ^{2}(2)-\frac{1}{2} G \log 2, \\
\sum_{n \geq 1} \frac{h_{2 n}}{n(2 n+1)}=\log 2+\frac{\pi}{2}-\frac{3}{4} \zeta(2) .
\end{gathered}
$$

For $a=-1$,

$$
\begin{aligned}
\int_{0}^{1} \frac{1}{x} \arctan ^{3}(x) d x & =\sum_{n \geq 1} \frac{(-1)^{n+1} h_{n}}{8 n^{2}}\left(\pi+H_{\frac{n}{2}-\frac{3}{4}}-H_{\frac{n}{2}-\frac{1}{4}}\right) \\
& =\frac{9}{8} \zeta(2) G+\frac{1}{1024}\left(\psi^{(3)}\left(\frac{3}{4}\right)-\psi^{(3)}\left(\frac{1}{4}\right)\right) \\
& =\frac{9}{8} \zeta(2) G-\frac{3}{2} \beta(4),
\end{aligned}
$$

where the Dirichlet function $\beta(4)=\sum_{j \geq 1} \frac{(-1)^{j+1}}{(2 j-1)^{4}}$. Here, we note that

$$
\psi^{(3)}\left(\frac{3}{4}\right)-\psi^{(3)}\left(\frac{1}{4}\right)=8 \pi^{4}-768 \beta(4)-\left(8 \pi^{4}+768 \beta(4)\right)=-1536 \beta(4) ;
$$


moreover, using the integral identity, we obtain the new Euler sum

$$
\begin{aligned}
\sum_{n \geq 1} \frac{(-1)^{n+1} h_{n}}{n^{2}}\left(H_{\frac{n}{2}+\frac{1}{4}}-H_{\frac{n}{2}-\frac{1}{4}}\right)= & 3 \zeta(2) G-12 \beta(4)+\frac{7}{4} \pi \zeta(3)+4 \pi G \\
& -7 \zeta(3)+8 G-3 \zeta(2)-2 \pi \log 2 .
\end{aligned}
$$

For the quartic

$$
\begin{aligned}
\int_{0}^{1} \frac{1}{x} \arctan ^{4}(x) d x & =\sum_{n \geq 1} \sum_{j \geq 1} \frac{(-1)^{n+j} h_{n} h_{j}}{j n(j+n)} \\
& =\frac{1}{16} \pi^{3} G+\frac{93}{32} \zeta(5)+\frac{3}{2} \pi \beta(4),
\end{aligned}
$$

and

$$
\begin{aligned}
\int_{0}^{1} x \arctan ^{6}(x) d x= & \sum_{n \geq 1} \sum_{j \geq 1} \sum_{k \geq 1} \frac{(-1)^{n+j+k+1} h_{n} h_{j} h_{k}}{2 j k n(j+k+n+1)} \\
= & \frac{15}{32} \pi^{3} G+\frac{20925}{1024} \zeta(5)-\frac{45}{4} \pi \beta(4)-\frac{3}{1024} \pi^{5} \\
& +\frac{945}{4096} \zeta(6)-\frac{675}{256} \zeta(4) \log 2+\frac{405}{256} \zeta(2) \zeta(3) .
\end{aligned}
$$

\section{Conclusions}

In the first three sections of the paper, we treated miscellaneous Euler sums, particularly, the alternating sums. We developed many new Euler type identities. In particular, we have developed some new identities for the Catalan constant, Apery's constant and Euler's famous $\zeta$ (2) constant. In the fourth section of this paper, we have demonstrated and explored the connection of integrals involving trigonometric and hyperbolic functions with Euler sums. We have evaluated particular integrals related to Euler sums, many of which are not amenable to a mathematical software package. Integrals dealing with powers of arctangent and hyperbolic functions will be further developed in a forthcoming paper.

Author Contributions: Conceptualization, A.S. and A.S.N.; methodology, A.S and A.S.N.; software, A.S.N.; validation, A.S.; formal analysis, A.S. and A.S.N.; investigation, A.S. and A.S.N.; writing-original draft preparation, A.S.N.; writing-review and editing, A.S.; visualization, A.S.N.; supervision, A.S.; project administration, A.S. and A.S.N.

Funding: This research received no external funding.

Conflicts of Interest: The authors declare no conflict of interest.

\section{References}

1. Lemmermeyer, F.; Mattmüller, M. (Eds.) Correspondence of Leonhard Euler with Christian Goldbach: Part I; Springer: Basel, Switzerland, 2015.

2. Euler, L. Meditationes circa singulare serierum genus. Novi Comment. Acad. Sci. Petropolitanae 1776, 20, 140-186. Available online: http:/ / eulerarchive.maa.org/ (accessed on 16 July 2019).

3. Nielsen, N. Handbuch der Theorie der Gammafunktion; Reprinted by Chelsea Publishing Company, Bronx, New York, 1965; Druck und Verlag von B. G. Teubner: Leipzig, Germany, 1906.

4. Sitaramachandrarao, R. A Formula of S. Ramanujan. J. Number Theory 1987, 25, 1-19. [CrossRef]

5. Georghiou, C.; Philippou, A.N. Harmonic Sums and the Zeta Function. Fibonacci Quart. 1983, 21, $29-36$.

6. Doelder, P.J.D. On some series containing $\psi(x)-\psi(y)$ and $(\psi(x)-\psi(y))^{2}$ for certain values of $x$ and $y$. J. Comput. Appl. Math. 1991, 37, 125-141. [CrossRef] 
7. Bailey, D.H.; Borwein, J.M.; Girgensohn, R. Experimental Evaluation of Euler Sums. Exp. Math. 1994, 3, 17-30. [CrossRef]

8. Borwein, D.; Borwein, J.M. On an intriguing integral and some series related to Z(4). Proc. Am. Math. Soc. 1995, 123, 1191-1198.

9. Borwein, D.; Borwein, J.M.; Girgensohn, R. Explicit Evaluation of Euler Sums. Proc. Edinburgh Math. Soc. 1995, 38, 277-294. [CrossRef]

10. Crandall, R.E.; Buhler, J.P. On the evaluation of Euler sums. Exp. Math. 1994, 3, 275-285. [CrossRef]

11. Sandham, H.F. Advanced Problem 4305. Am. Math. Mon. 1948, 55, 431. [CrossRef]

12. Chu, W. Hypergeometric series and the Riemann zeta function. Acta Arith. 1997, LXXXII.2, $104-118$. [CrossRef]

13. Jung, M.; Cho, Y.J.; Choi, J. Euler Sums Evaluatable From Integrals. Commun. Korean Math. Soc. 2004, 19, 545-555. [CrossRef]

14. Lewin, L. Polylogarithms and Associated Functions; Title of Original 1958 Edition: Dilogarithms and Associated Functions; Elsevier: North Holland, NY, USA, 1981.

15. Flajolet, P.; Salvy, B. Euler Sums and Contour Integral Representations. Exp. Math. 1998, 7, 15-35. [CrossRef]

16. Freitas, P. Integrals of Polylogarithmic Functions, Recurrence Relations, and Associated Euler Sums. Math. Comp. 2005, 74, 1425-1440. [CrossRef]

17. Berndt, B.C. Ramanujan's Notebooks Part I; Springer: New York, NY, USA, 1985.

18. Choi, J. Log-sine and log-cosine integrals. Honam Math. J. 2013, 35, 137-146. [CrossRef]

19. Ciaurri, O.; Navas, L.M.; Ruiz, F.J.; Varona, J.L. A simple computation of $\zeta(2 k)$ by using Bernoulli polynomials and a telescoping series. arXiv 2013, arXiv:1209.5030.v2.

20. Shang, Y. A class of combinatorial functions for Eulerian numbers. Matematika 2012, 28, 151-154.

21. Sofo, A. General order Euler sums with rational argument. Integral Transform. Spec. Funct. 2019. [CrossRef]

22. Choi, J.; Srivastava, H.M. Series involving the Zeta functions and a family of generalized Goldbach-Euler series. Am. Math. Mon. 2014, 121, 229-236. [CrossRef]

23. Choi, J.; Srivastava, H.M. Some applications of the gamma and polygamma functions involving convolutions of the Rayleigh functions, multiple Euler sums and log-sine integrals. Math. Nachr. 2009, 282, 1709-1723. [CrossRef]

24. Chu, W.; Esposito, F.L. Evaluation of Apéry-like series through multisection method. J. Class. Anal. 2018, 12, $55-77$.

25. Ryotaro, H. On Euler's formulae for double zeta values. Kyushu J. Math. 2018, 72, 15-24.

26. Li, A.; Qin, H. The representation of the generalized linear Euler sums with parameters. Integral Transform. Spec. Funct. 2019, 30, 656-681. [CrossRef]

27. Liu, H.; Wang, W. Gauss's theorem and harmonic number summation formulae with certain mathematical constants. J. Differ. Equ. Appl. 2019, 25, 313-330. [CrossRef]

28. Mező, I. A family of polylog-trigonometric integrals. Ramanujan J. 2018, 46, 161-171. [CrossRef]

29. Petojević, A.; Srivastava, H.M. Computation of Euler's type sums of the products of Bernoulli numbers. Appl. Math. Lett. 2009, 22, 796-801. [CrossRef]

30. Sofo, A. Shifted harmonic sums of order two. Commun. Korean Math. Soc. 2014, 29, 239-255. [CrossRef]

31. Sofo, A. New classes of harmonic number identities. J. Integer Seq. 2012, 15, 12.

32. Sofo, A.; Cvijović, D. Extensions of Euler harmonic sums. Appl. Anal. Discret. Math. 2012, 6, 317-328. [CrossRef]

33. Sofo, A.; Srivastava, H.M. A family of shifted harmonic sums. Ramanujan J. 2015, 37, 89-108. [CrossRef]

34. Teo, L.P. Alternating double Euler sums, hypergeometric identities and a theorem of Zagier. J. Math. Anal. Appl. 2018, 462, 777-800. [CrossRef]

35. Wang, W.; Lyu, Y. Euler sums and Stirling sums. J. Number Theory 2018, 185, 160-193. [CrossRef]

36. Wei, C.; Wang, X. Whipple-type ${ }_{3} F_{2}$ series and summation formulae involving generalized harmonic numbers. Int. J. Number Theory 2018, 14, 2385-2407. [CrossRef]

37. Xu, C.; Cai, Y. On harmonic numbers and nonlinear Euler sums. J. Math. Anal. Appl. 2018, 466, 1009-1042. [CrossRef]

38. Gradshteyn, I.S.; Ryzhik, I.M. Table of Integrals, Series, and Products; Academic Press: New York, NY, USA, 2007.

39. Bromwich, T.J. An Introduction to the Theory of Infinite Series; Macmillan: London, UK, 1908.

40. Ferrar, W.L. A Text-Book of Convergence; Oxford University Press: Oxford, UK, 1938. 
41. Knopp, K. Theory and Application of Infinite Series; Blackie \& Son: New York, NY, USA, 1951.

42. Sofo, A. Evaluation of integrals with hypergeometric and logarithmic functions. Open Math. 2018, 16, 63-74.

43. Sofo, A. General order Euler sums with multiple argument. J. Number Theory 2018, 189, 255-271. [CrossRef]

44. Nimbran, A.S.; Sofo, A. New Interesting Euler Sums. J. Class Anal. 2019, Accepted for publication in August.

(C) 2019 by the authors. Licensee MDPI, Basel, Switzerland. This article is an open access article distributed under the terms and conditions of the Creative Commons Attribution (CC BY) license (http:/ / creativecommons.org/licenses/by/4.0/). 